\title{
Systematic Approach to Optimization for Protection Against Intentional Ultrashort Pulses Based on Multiconductor Modal Filters
}

\author{
Anton O. Belousov $(\mathbb{D})$ and Talgat R. Gazizov $(\mathbb{D}$ \\ Department of Television and Control, Tomsk State University of Control Systems and Radioelectronics, Tomsk, Russia \\ Correspondence should be addressed to Anton O. Belousov; ant1lafleur@gmail.com
}

Received 31 December 2017; Revised 9 April 2018; Accepted 18 April 2018; Published 5 June 2018

Academic Editor: Kevin Wong

Copyright ( 2018 Anton O. Belousov and Talgat R. Gazizov. This is an open access article distributed under the Creative Commons Attribution License, which permits unrestricted use, distribution, and reproduction in any medium, provided the original work is properly cited.

\begin{abstract}
The problem of protecting radio electronic equipment from ultrashort pulses is of utmost importance nowadays since conductive interference poses the biggest danger to its proper functioning. The article considers the issue of protecting equipment by means of modal filters (MFs) and analyzes the structures of multiconductor microstrip MFs. We present the results of a complex study of the possibility to conduct the optimization (both separate and simultaneous) of a multiconductor MF by different criteria and the formulation of the basic (electrical) optimization criteria for MF. We have formulated the amplitude and time criteria for optimizing an MF (with any number of conductors) in an analytical form and obtained a general multicriteria objective function for optimizing an MF by different criteria. As a result, we have formed a hybrid model consisting of heuristic search and GA. The results demonstrated the topicality of further research in this field.
\end{abstract}

This study has been carried out in memory of the authors' teacher Ivan Nikolaevich Pustynsky who died on 29.12.2017

\section{Introduction}

Contemporary radioelectronic equipment has huge functionality but, at the same time, is susceptible to electromagnetic interference. Conducted interference is considered the most harmful one, as it can penetrate into devices directly through conductors [1]. Modern generators of ultrashort pulses have very high capabilities [2]. Such ultrashort pulses are able to penetrate and disturb electronics due to the high power output and short duration. Therefore, it is necessary to improve the protection of electronic equipment against ultrashort pulses.

One of the new protection principles is based on modal filtering-the use of modal distortions (signal changes due to the difference in the mode delay of a multiconductor transmission line (MCTL)) due to the serial modal decomposition of the pulse in segments of coupled lines. A series of researches performed indicate the possibility of creating protection devices based on modal filtering-modal filters (MF) [3]. They can have such benefits as radiation resistance, low mass, and costeffectiveness. As a device for protection against pulse disturbances, you can use a strip structure on a widespread foil-coated glass fiber sheet [4]. However, previously, there were studied MFs based only on a pair of coupled lines, while MFs on multiconductor lines are almost unexplored. Thus, the use of MCTL resources in the MF is relevant.

The creation of any new equipment often requires modeling complex systems and solving complex simulation problems. For this aim, there are successfully used optimization methods based on evolutionary algorithms, for example, in power electronics [5] and applied electrodynamics [6]. Unfortunately, a number of trends in developing devices for protection against ultrashort pulses are also increasingly reduced to simulating complex systems.

Indeed, first of all, the number of optimized parameters increases, which in the case of MF is determined by the increase in the number of conductors and dielectrics, as well 
as the complexity of their geometry. A significant contribution to the complexity is made by the expansion of the parameter range, as well as the decrease in the step of the optimized parameter, determined by the complex behavior of the characteristics of interest. When optimizing an MF for the parameters, the parameters include not only the conductors and dielectrics of the MF but also the exciting signals. These signals are divided into two large groups: useful and noise. The complexity of the solution can dramatically increase if you optimize not only the parameters, but also the very structure as well. Finally, the convergence of optimization can deteriorate with the growth of the number of simultaneously satisfied criteria (electrical, mass size, and cost) of optimization. Thus, the search for new approaches to reducing computational complexity in the optimization becomes relevant.

The research of separate issues of multiconductor MF optimization has already been carried out [7-11]. However, there is no systematic representation of the recently obtained and new results on solving the complex problem of creating new devices for protecting against ultrashort pulses in a single work. Meanwhile, it is seen important for a wide range of designers of critical electronics. The aim of this paper is to fill this gap. Therefore, it is useful to carefully investigate the propagation of signals in multiconductor MFs and also to formulate the basic criteria for optimizing their parameters. We prefer to start it with the preliminary simulation and optimization of the parameters by heuristic search as it is the simplest and most widely used method of optimization. Further, to validate the results obtained earlier, it is useful to do optimization using the genetic algorithm (GA) as the most popular algorithm for optimization in the problems of electrodynamics and propagation of radio waves [6]. Then, it is expedient to execute the formulation of the basic optimization criteria in an analytical form and proceed from one criterion to multicriteria optimization.

\section{Structures and Schematic Diagrams of MFs under Consideration}

As the object of investigation, we took multiconductor microstrip lines (MSLs) of lengths $l=400,100$, and $60 \mathrm{~cm}$, consisting of 1-5 conductors. (The length of $400 \mathrm{~cm}$ was chosen in the first stage of the investigation to ensure the complete decomposition of the pulse signal in the simulation without considering losses. The lengths of 100 and $60 \mathrm{~cm}$ were chosen at the subsequent stages of the investigation for preliminary simulation, which took losses in conductors and dielectrics into account, and for further experimental realization.) The cross sections of these lines are generally shown in Figure 1 where $w$ is the width of conductors, $s_{i}$ is separations between them, $t$ is the thickness of conductors and $h$ is the thickness of the dielectric, and $\varepsilon_{r}$ is the permittivity of the dielectric. The schematic diagrams of these MFs are shown in Figure 2.

First, we constructed geometric models of the MSL cross section. Then, we calculated the matrixes of per-unit-length coefficients of electrostatic $\mathbf{C}$ and electromagnetic $\mathbf{L}$ induction. If it is necessary to take into account the losses, we calculated the matrixes of the per-unit-length resistances $\mathbf{R}$ (for the losses in the conductors) and conductivities $\mathbf{G}$ (for the losses in the dielectrics). When considering the losses, we used a widely known model [12] of the frequency dependence of the relative permittivity and tangent of the dielectric loss angle of FR-4 material for calculating the $\mathbf{G}$ matrix. The entries of the $\mathbf{R}$ matrix were calculated taking into account the skin effect, the proximity effect, and losses in the ground plane using the method proposed in [13]. Next, we drew a schematic diagram for simulation, set loads and pulse excitation values, computed time response to the excitation in the parameter range, and optimized parameters of a MSL.

It was assumed that a $\mathrm{T}$ wave is propagating along the considered lines. The parameters and forms of the signal were calculated in TALGAT software [14], wherein the abovementioned models and steps were implemented. As the exciting pulses, we used a trapezoidal pulse with rise, fall, and flat top durations of $50 \mathrm{ps}$ (the overall duration of $150 \mathrm{ps,}$ to approximate the typical ultrashort pulse in the simulation without considering losses) with an amplitude of $5 \mathrm{~V}$ and a digitized signal of the S9-11 oscilloscope (for the maximum possible approximation to the actual exciting signal in the simulation considering losses) with an amplitude of $0.644 \mathrm{~V}$ (measured at $50 \Omega$ load) and the durations of rise of $56 \mathrm{ps}$, fall of $48 \mathrm{ps}$, and flat top of $4 \mathrm{ps}$ (the overall duration was $108 \mathrm{ps}$; durations were measured at levels of 0.1-0.9). The waveforms of the exciting pulses are shown in Figure 3.

\section{Formulation of Optimization Criteria}

Optimization can be carried out by various criteria. The criteria considered below allow us to obtain higher characteristics of a multiconductor MF for protection against ultrashort pulses.

(1) Minimization of the maximum voltage of an MF output waveform:

$$
\max (U(t)) \rightarrow \min .
$$

This criterion is the most important one, as signal amplitude at the output of an MF defines its main characteristic: filter attenuation. However, depending on the defined excitation, optimization by this criterion can give various results. It also requires time-consuming computation of the time-domain response that complicates the optimization of complex structures.

(2) Equalization of decomposition pulse delay differences:

$$
\min \left|t_{i+1}-t_{i}\right| \rightarrow \max , \quad i=1, \ldots, N p-1,
$$

where $N p$ is the number of decomposition pulses, $t_{i}$ is the value of the $i$ th pulse delay.

This criterion is important, as it increases the maximum duration of an initial pulse, which is completely decomposed, and prevents the pulse 


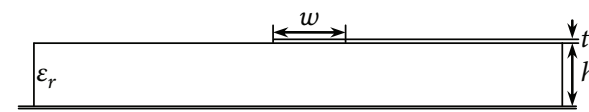

(a)

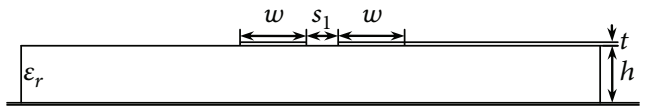

(b)

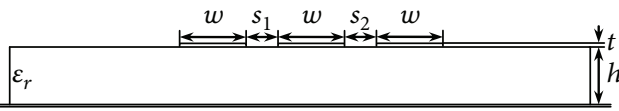

(c)

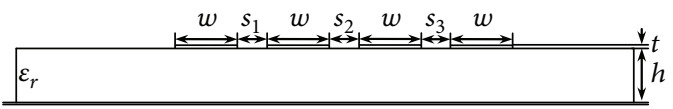

(d)

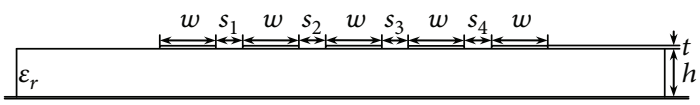

(e)

Figure 1: Cross sections of one- (a), two- (b), three- (c), four- (d), and five- (e) conductor structures.

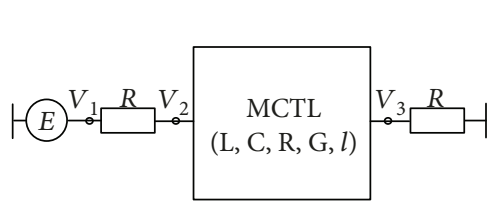

(a)

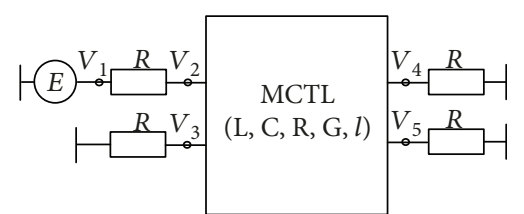

(b)

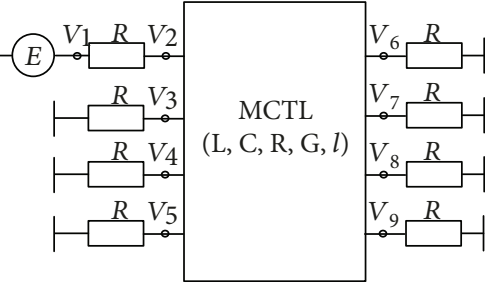

(d)

(c)

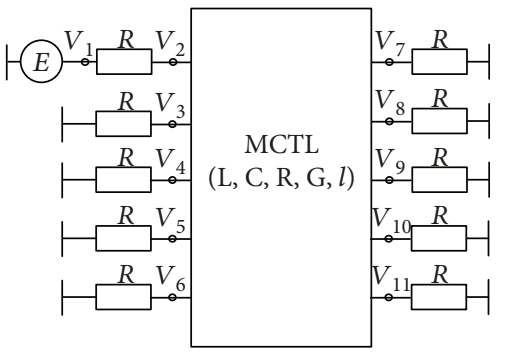

(e)

FIGURE 2: Schematic diagrams for one- (a), two- (b), three- (c), four- (d), and five- (e) conductor structures.

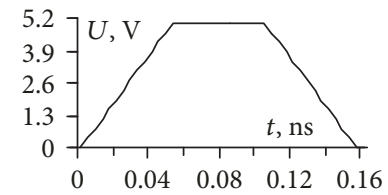

(a)

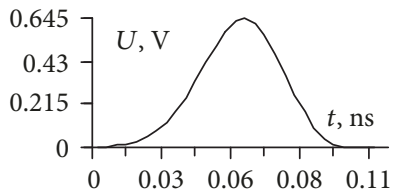

(b)

FIGURE 3: EMF waveforms for the overall duration of $150 \mathrm{ps}$ (a) and $108 \mathrm{ps}$ (b).

overlapping, which increases the maximum voltage at the line output.

(3) Maximization of difference between the maximum and the minimum pulse delays:

$$
\left(t_{\max }-t_{\min }\right) \rightarrow \max
$$

where $t_{\max }$ and $t_{\min }$ are the maximum and the minimum values of pulse delays, respectively.

This criterion is important, as it additionally increases the maximum duration of an initial pulse, which is completely decomposed. Let us note that in this formulation the criterion can give decomposition pulses in any 
TABle 1: Peak voltage of decomposition pulses, V.

\begin{tabular}{|c|c|c|c|c|c|c|c|c|c|c|c|c|}
\hline \multirow{2}{*}{$\begin{array}{l}\text { Type of result } \\
\text { Analytics }\end{array}$} & \multicolumn{3}{|c|}{$\begin{array}{c}N=3\left(s_{1}=400 \mu \mathrm{m}\right. \\
\left.s_{2}=540 \mu \mathrm{m}\right)\end{array}$} & \multicolumn{4}{|c|}{$\begin{array}{c}N=4\left(s_{1}=500 \mu \mathrm{m}, s_{2}=675 \mu \mathrm{m}\right. \\
\left.\quad \text { and } s_{3}=650 \mu \mathrm{m}\right)\end{array}$} & \multicolumn{5}{|c|}{$\begin{array}{c}N=5\left(s_{1}=367 \mu \mathrm{m}, s_{2}=447 \mu \mathrm{m}, s_{3}=500 \mu \mathrm{m},\right. \\
\left.\text { and } s_{4}=685 \mu \mathrm{m}\right)\end{array}$} \\
\hline & 0.84 & 0.97 & 0.67 & 0.61 & 0.74 & 0.64 & 0.44 & 0.52 & 0.66 & 0.60 & 0.44 & 0.23 \\
\hline Simulation & 0.84 & 0.83 & 0.83 & 0.70 & 0.49 & 0.70 & 0.60 & 0.56 & 0.47 & 0.39 & 0.56 & 0.49 \\
\hline
\end{tabular}

part of a time axis, while the increase of a delay of all pulses can be adverse. In order to minimize it, it is possible to use other formulations:

$$
\begin{gathered}
\left(\frac{t_{\min }-l}{c}\right) \rightarrow \min , \\
\left(\frac{\varepsilon_{r_{\max }}{ }^{0.5} l}{c-t_{\max }}\right) \rightarrow \min ,
\end{gathered}
$$

where $l$ is the length of an MF, c is light velocity in vacuum, and $\varepsilon_{r \text { max }}$ is the maximum value of $\varepsilon_{r}$ of MF dielectrics.

The criterion (4) does make the first pulse delay as short as possible, that is, as determined by light velocity in vacuum. The criterion (5) makes the last pulse delay as long as possible, that is, determined by light velocity in dielectric with the maximum value of $\varepsilon_{r}$.

\section{Accelerated Calculation of Objective Function}

Minimization of $\max (U(t))$ demands simulation of a timedomain response. However, it can be computationally expensive, and, therefore, its acceleration is relevant. Meanwhile, while matching the modes of a multiconductor transmission line, amplitudes of decomposition pulses can be computed using analytical expression [6].

$$
\mathbf{V}=\mathbf{S}_{v} \operatorname{diag}\left(\mathbf{V}_{m}\right),
$$

where $\mathbf{V}_{m}=0.5 \mathbf{S}_{v}{ }^{-1} \mathbf{E} ; \mathbf{S}_{v}$ is a matrix of $N \times N$ size that contains eigenvectors of $\mathbf{L C}$ matrix; $\mathbf{E}$ is a vector of $N \times 1$ size that consists of the values of voltage source amplitudes, where $N$ is a number of conductors.

As for maximization of $\min \left|t_{i+1}-t_{i}\right|$ and maximization of $\left(t_{\max }-t_{\min }\right)$, generally, they also require calculation of a time-domain response, as we need the values of the moments when decomposition pulses arrive at the end of the line. However, practical experience in simulating an MF shows that for the matched MF and weak coupling between the conductors, the maximum amplitude of pulses is defined by amplitudes of the first traveling wave pulses (subjected to one reflection at most). The moments of their arrival to the end of MF are defined by values of per-unit-length delay of modes. In such case, for optimization, there is no need to calculate a time-domain response, and it is enough to calculate only per-unit-length delay that considerably reduces computational costs. Optimization criteria are slightly changed. So, (2) takes a form of

$$
\min \left|\tau_{i+1}-\tau_{i}\right| \rightarrow \max , \quad i=1, \ldots, N-1,
$$

where $N$ is the number of conductors, $\tau_{i}$ is the value of per-unit-length delay of the $i$ th pulse. From (3), we obtain

$$
\left(\tau_{\max }-\tau_{\min }\right) \rightarrow \max
$$

where $\tau_{\max }$ and $\tau_{\min }$ are the maximum and the minimum values of per-unit-length delay. From (4) and (5), we obtain

$$
\begin{aligned}
& \left(\frac{\tau_{\min }-1}{\mathrm{c}}\right) \rightarrow \min , \\
& \left(\frac{\varepsilon_{r_{\max }}^{0.5}}{c-\tau_{\max }}\right) \rightarrow \min .
\end{aligned}
$$

Thus, the presented analytical expressions can considerably accelerate calculation of objective function.

\section{MF Optimization by Heuristic Search}

5.1. Optimization by Criterion of Minimizing the Maximum Voltage at the MF Output. To begin with, we can optimize the parameters by using heuristic search for MF with $N=2$, $3,4,5$ by the criterion of minimizing the maximum amplitude of the output signal. Losses in conductors and dielectrics were not taken into account. As for the exciting pulses, we used signal shown in Figure 3(a).

For $N=2$, the absolute value of difference of modal perunit-length delays is $0.34 \mathrm{~ns} / \mathrm{m}$ at $s=500 \mu \mathrm{m}, w=290 \mu \mathrm{m}$, $t=105 \mu \mathrm{m}, h=190 \mu \mathrm{m}$ (standard material), and $\varepsilon_{r}=5$. The value of $w$ was optimized, in order to assure characteristic impedance of a single line to be equal to $50 \Omega$, and it was kept unchanged thereafter, just as the values of $t, h$, and $\varepsilon_{r}$. For $N=2$, we took the typical values of the parameters $s, t, h$, and $\varepsilon_{r}$ for the technology of printed circuit boards. The length of the line was $400 \mathrm{~cm}$. Each pair of neighboring conductors has its own gap between the conductors $\left(s_{i}\right)$, and these values were optimized in the range of $1-1000 \mu \mathrm{m}$ to equalize delay differences between decomposition pulses at the output of an active conductor of the MSL.

Generally, 3, 4, and 5 modes are propagating along the line with $N=3,4,5$ correspondingly, and each mode has its own characteristics. The optimized values of $s_{i}$ for $N=3,4$, 5 , as well as the values of the amplitudes of the decomposition pulses, calculated analytically and by simulation with the EMF source with the amplitude of $5 \mathrm{~V}$ connected between conductor 1 (active) and the reference conductor, are presented in Table 1. The voltage waveforms at the input and output of the lines are shown in Figure 4. One can see 3, 4, and 5 pulses with maximum amplitudes, which are by 3 , 3.6, and 4.5 times, respectively, less than the signal level at the beginning of the line. Thus, adding more conductors with identical parameters to the two-conductor structure, with identical parameters, significantly (almost by 5 times) decreases the amplitude at the line output. 


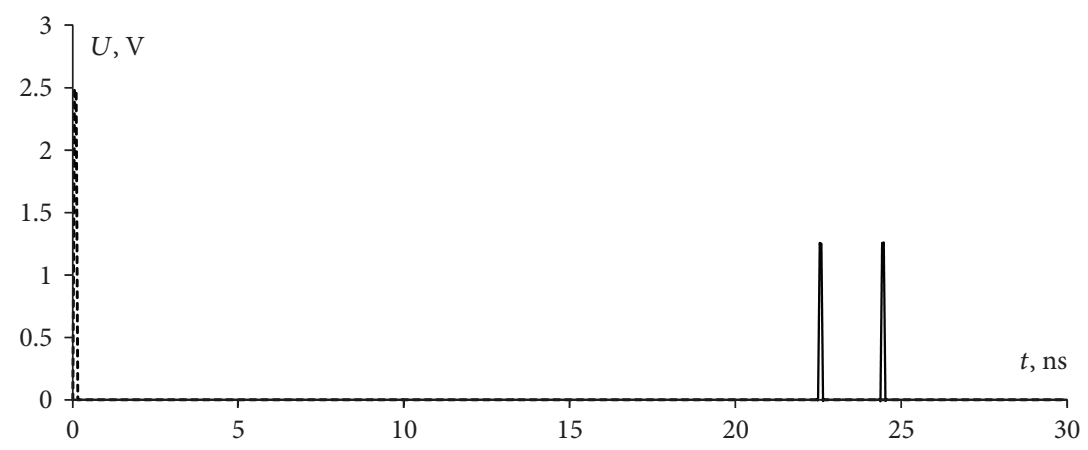

(a)

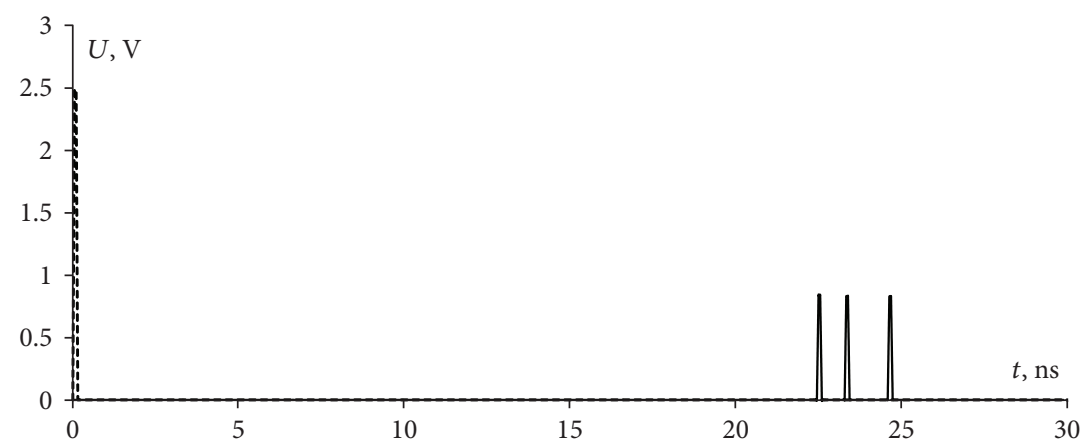

(b)

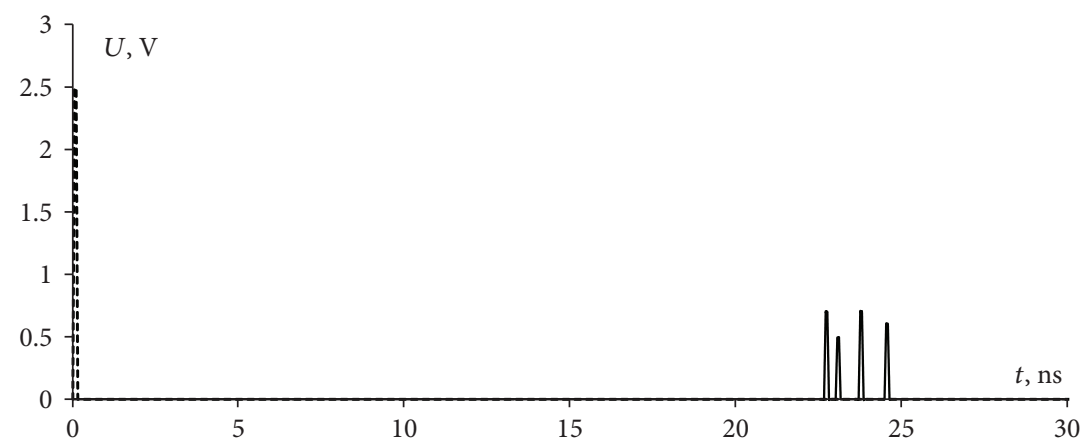

(c)

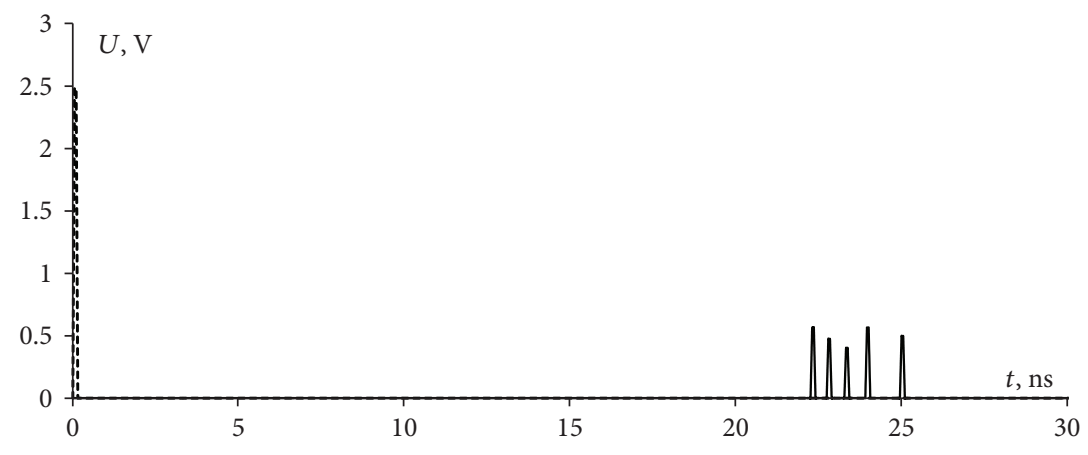

(d)

Figure 4: Voltage waveforms at the input (---) and the output (-) of conductor 1 for two- (a), three- (b), four- (c), and five- (d) conductor MFs with parameters, obtained by heuristic search optimization.

5.2. Optimization by Criterion of Equalizing the Time Intervals between the Decomposition Pulses. Figure 4 shows a decrease in the pulse amplitude at the MF output with increasing $N$. However, the difference of the maximum and minimum delays has remained unaddressed that has led to an uncontrollable variation of this value in different structures and, as a result, has limited the maximum duration of the initial pulse which will be decomposed completely. 
TABLE 2: Values of $s_{i}$ and per-unit-length delays after optimization using a heuristic search.

\begin{tabular}{|c|c|c|c|c|c|c|c|c|c|c|c|c|}
\hline \multirow{2}{*}{$\begin{array}{l}\text { Parameter } \\
\tau_{i}, \mathrm{~ns} / \mathrm{m}\end{array}$} & \multicolumn{3}{|c|}{$\begin{array}{c}N=3\left(s_{1}=170 \mu \mathrm{m}\right. \\
\left.s_{2}=540 \mu \mathrm{m}\right)\end{array}$} & \multicolumn{4}{|c|}{$\begin{array}{c}N=4\left(s_{1}=70 \mu \mathrm{m}, s_{2}=335 \mu \mathrm{m}\right. \\
\left.\text { and } s_{3}=250 \mu \mathrm{m}\right)\end{array}$} & \multicolumn{5}{|c|}{$\begin{array}{l}N=5\left(s_{1}=15 \mu \mathrm{m}, s_{2}=32 \mu \mathrm{m}\right. \\
\left.s_{3}=290 \mu \mathrm{m}, \text { and } s_{4}=200 \mu \mathrm{m}\right)\end{array}$} \\
\hline & 5.37 & 5.81 & 6.25 & 5.02 & 5.49 & 5.96 & 6.43 & 4.2 & 4.8 & 5.4 & 6 & 6.6 \\
\hline
\end{tabular}

Obviously, when applying the pulse, with a total duration of more than $200 \mathrm{ps,}$, with the same line parameters, we will observe the overlapping of the pulses, and as a consequence, an increase in the amplitude of the signal at the end of the line. Thus, it is also relevant to make optimization by another criterion-equalization of decomposition pulse delay differences.

The parameters $s_{i}$ (with the previous values of the remaining parameters) were optimized by the specified criterion using heuristic search for MFs with $N=3,4,5$. Losses in conductors and dielectrics were not taken into account.

The optimized values of $s_{i}$, as well as the per-unit-length modal delays, are given in Table 2 . All values of $s_{i}$ are significantly different from the values obtained by the criterion of amplitude decrease, because equalization of delay differences between decomposition pulses is achieved by means of increasing the coupling between the external (active) and central conductors.

Table 3 gives the values of the maximum amplitudes of decomposition pulses at the end of the active conductor for $N=3,4,5$ for different optimization criteria computed with (6) analytically $\left(\max \left(\mathbf{V}_{\mathbf{A}}\right)\right)$ and according to the time response $\left(\max \left(\mathbf{V}_{\mathbf{R}}\right)\right)$, as well as the minimum values of differences of modal per-unit-length delays.

It is clear from Table 3 that when equalization of absolute values of differences of modal per-unit-length delays $\left(\Delta \tau_{i}\right)$ is taken as an optimization criterion, the value of $\min \left(\Delta \tau_{i}\right)$ increased by 1.4 times simultaneously with the increase of $N$, and, in comparison with the optimization that took $\max (\mathbf{V})$ as an optimization criterion, it increased by 2 times for $N=3$ and by 5 times for $N=4$ and $N=5$.

However, improvement of one parameter leads to degradation of another. As shown in Table 3, the maximum amplitudes of decomposition pulses are 2.2, 2.3, and 3.9 times less than the pulse amplitude at the near end of the line, which is less than the corresponding values $(3,3.6$, and 4.5$)$ for optimization that take amplitudes of an output signal as an optimization criterion.

There is a tendency for increasing the difference between the values of the maximum amplitudes of decomposition pulses, calculated analytically and according to the response. This tendency appears when the number of $N$ starts to grow and is the strongest for $N=5$. It can be explained by incomplete matching of a transmission line. However, the very fact that the maximum amplitudes of decomposition pulses have been accurately computed using (6) is rather important; as in the future, it provides fast optimization by using an analytical formula, that is, it will not be necessary to compute time response.

Apparently, different results can be obtained for different aims. For example, in optimization by equalizing decomposition pulse delay differences, central conductors are seen to be
TABLE 3: Results of MF optimization for $N=3,4,5$ separately by two criteria.

\begin{tabular}{lcccccc}
\hline Criterion & \multicolumn{3}{c}{$\max (U(t)) \rightarrow \min$} & \multicolumn{3}{c}{$\min \left|\tau_{i+1}-\tau_{i}\right| \rightarrow \max$} \\
$N$ & 3 & 4 & 5 & 3 & 4 & 5 \\
\hline $\min \left(\Delta \boldsymbol{\tau}_{\boldsymbol{i}}\right), \mathrm{ns} / \mathrm{m}$ & 0.21 & 0.09 & 0.12 & 0.44 & 0.47 & 0.6 \\
$\max \left(\mathbf{V}_{\mathbf{A}}\right), \mathrm{V}$ & 0.97 & 0.74 & 0.66 & 1.16 & 1.15 & 0.9 \\
$\max \left(\mathbf{V}_{\mathbf{R}}\right), \mathrm{V}$ & 0.84 & 0.70 & 0.56 & 1.14 & 1.08 & 0.65 \\
\hline
\end{tabular}

closer to external ones, but this optimization does not give the minimum level of ultrashort pulse at the line output.

As we have already mentioned above, unaccounted delay differences between pulses can cause incomplete decomposition of a pulse and, at the same time, the increase in its duration, and, consequently, it can cause overlapping of pulses that leads to the increase of pulse amplitudes at the end of a line. But if the overlapped pulses together do not exceed the maximum amplitude of the rest pulses, then this overlapping can be considered not crucial.

\subsection{Optimization by Criterion of Maximizing the Difference} between the Maximum and the Minimum Pulse Delays. Note that previously the difference of the maximum and minimum delays has remained unaddressed, which has led to an uncontrollable variation of its value in different structures and, as a result, has limited the maximum duration of an initial pulse that will be decomposed completely. Thus, it is also relevant to conduct optimization by another criterion-maximization of the difference between the maximum and the minimum pulse delays.

The parameters $s_{i}$ (with the previous values of the remaining parameters) were optimized by the specified criterion using heuristic search for $\mathrm{MF}$ for $N=3,4,5$. The losses in conductors and dielectrics were not taken into account. The MFs were simulated with $w=290 \mu \mathrm{m}, t=105 \mu \mathrm{m}$, $h=190 \mu \mathrm{m}$, and $\varepsilon_{r}=5$. As before, the parameters $s_{i}$ were optimized. For $N=3$, we considered two values of the lower limit of range $s: 50$ and $1 \mu \mathrm{m}$. The first was taken, for practical reasons, as the minimum for printed circuit boards. The second was taken to assess limit values of the characteristics of interest.

Table 4 shows the optimization results for $N=3,4,5$ separately by three different criteria: minimization of $\max (U(t))$, maximization of $\min \left|t_{i+1}-t_{i}\right|$, and maximization of $\left(t_{\max }-t_{\text {min }}\right)$.

The maximum amplitudes of pulses at the output of MF are calculated by (6) and represented in the last line of Table 4 . As you can see, they are very close to the amplitudes obtained from a response. (The exception is the last case of a very close coupling, for which an error of expression (6) is too high, as it does not consider reflection of modes.) Thus, 
TABLE 4: Results of MF optimization for $N=3,4,5$ using different criteria.

\begin{tabular}{lcccccccccc}
\hline Criterion & \multicolumn{3}{c}{$\max (U(t)) \rightarrow \min$} & \multicolumn{3}{c}{$\min \left|t_{i+1}-t_{i}\right| \rightarrow \max$} & \multicolumn{3}{c}{$\left(t_{\max }-t_{\min }\right) \rightarrow \max$} \\
$N$ & 3 & 4 & 5 & 3 & 4 & 5 & 3 & 5 \\
\hline$s_{i}, \mu \mathrm{m}$ & 400,540 & $500,675,650$ & $367,447,500,685$ & 170,540 & $70,335,250$ & $15,32,290,200$ & 50,50 & 1,10 & $50,50,50$ & $50,50,50,50$ \\
$\tau_{\max }-\tau_{\min }$, & 0.534 & 0.45 & 0.67 & 0.88 & 1.4 & 2.39 & 1.78 & 3 & 1.98 & 2.11 \\
$\mathrm{~ns} / \mathrm{m}$ & & & & & & & & & & \\
$t_{\max }-t_{\min }, \mathrm{ns}$ & 2.136 & 1.8 & 2.68 & 3.52 & 5.6 & 9.56 & 7.12 & 12 & 7.92 & 8.44 \\
$\max \left(\mathbf{V}_{\mathbf{R}}\right), \mathrm{V}$ & 0.842 & 0.70 & 0.56 & 1.14 & 1.08 & 0.65 & 1.22 & 0.8 & 1.05 & 0.87 \\
$\max \left(\mathbf{V}_{\mathbf{A}}\right), \mathrm{V}$ & 0.838 & 0.70 & 0.56 & 1.16 & 1.16 & 0.68 & 1.25 & 1.27 & 1.06 & 0.88 \\
\hline
\end{tabular}

TABLE 5: Results of the GA optimization of $s_{1}(\mu \mathrm{m})$ by minimizing the amplitude of the signal $(\max (U(t))(\mathrm{V}))$ at the three-conductor MF output.

\begin{tabular}{|c|c|c|c|c|c|c|c|c|}
\hline \multirow{3}{*}{$N$} & \multicolumn{8}{|c|}{ Number of individuals, number of generations } \\
\hline & \multicolumn{2}{|c|}{3,10} & \multicolumn{2}{|c|}{10,10} & \multicolumn{2}{|c|}{10,30} & \multicolumn{2}{|c|}{10,100} \\
\hline & $s_{1}$ & $\max (U(t))$ & $s_{1}$ & $\max (U(t))$ & $s_{1}$ & $\max (U(t))$ & $s_{1}$ & $\max (U(t))$ \\
\hline 1 & 366 & 0.0373535 & 333 & 0.0364478 & 323 & 0.0366652 & 329 & 0.0364521 \\
\hline 2 & 264 & 0.0387915 & 332 & 0.0364339 & 325 & 0.0365686 & 330 & 0.0364266 \\
\hline 3 & 273 & 0.0384555 & 328 & 0.0364779 & 327 & 0.0364996 & 329 & 0.0364521 \\
\hline 4 & 345 & 0.0364985 & 325 & 0.0365686 & 327 & 0.0364996 & 330 & 0.0364266 \\
\hline 5 & 319 & 0.0368421 & 326 & 0.0365231 & 324 & 0.0366183 & 330 & 0.0364266 \\
\hline
\end{tabular}

these examples confirm the possibility of rapid optimization by an analytical formula, that is, without time-consuming response calculations.

For $s_{i} \geq 50 \mu \mathrm{m}$, the optimized values of $s_{i}$ are in the lower limit of the range $\left(s_{1}=s_{2}=50 \mu \mathrm{m}\right)$, and for $s_{i} \geq 1 \mu \mathrm{m}$, they are near it $\left(s_{1}=1 \mu \mathrm{m}, s_{2}=10 \mu \mathrm{m}\right)$. They considerably differ from the values obtained earlier, which are clear from the first line of Table 4 . For $N=3$ and $s_{1}=s_{2}=50 \mu \mathrm{m}$, the value $\left(\tau_{\max }-\tau_{\min }\right)$ has increased twice in comparison with the previous criterion, and for $s_{1}=1 \mu \mathrm{m}, s_{2}=10 \mu \mathrm{m}$, it is 1.7 times more additionally, and it was revealed that it is considerably influenced only by $s_{1}$. The value $\left(t_{\max }-t_{\min }\right)$ has also increased by 1.7 times. For $N=4$ and $s_{i}=50 \mu \mathrm{m}$, the value $\left(\tau_{\max }-\tau_{\min }\right)$ has increased by 1.4 times in comparison with the previous criterion, and for $N=5$, it has decreased by 1.1 times, and it was revealed that it is considerably influenced only by $s_{1}$, which value in the previous criterion was $15 \mu \mathrm{m}$ for $N=5$.

As you can see, the improvement of one parameter (the increase of delay difference) can worsen another (the increase of the maximum pulse amplitude). For example, when changing over from minimization of $\max (U(t))$ to maximization of $\left(t_{\max }-t_{\min }\right)$, the value of $\max (U(t))$ increases by 1.45 times for $N=3$, by 1.5 times for $N=4$, and by 1.55 times for $N=5$. However for $N=3$, when $s_{i}$ is reduced, the value of $\max (U(t))$ decreases to $0.8 \mathrm{~V}$, yielding the best result.

\section{Hybrid Model of MF Optimization by Heuristic Search and Genetic Algorithm}

Heuristic search of parameters was used in Section 5. This method was sufficient to achieve one or another criterion, but does not guarantee the best results. Therefore, it is better to use optimization techniques to improve earlier results. It is also indicative to compare the obtained results with the results of heuristic search.

6.1. Optimization by Heuristic Search. In Section 5.1, the optimization of the three-conductor MF by heuristic search has been carried out; however, in this Section, in order to be closer to real conditions, we used a real initial pulse (Figure 3(b)) with losses in the conductors and dielectrics being taken into account. Thus, to obtain consistent comparison results, it is necessary to perform the optimization by heuristic search once again.

The MF was optimized for $w=1000 \mu \mathrm{m}, t=18 \mu \mathrm{m}$, $h=500 \mu \mathrm{m}, \varepsilon_{r}=4.5$, and $l=60 \mathrm{~cm}$. The value of $w$ was preliminarily optimized in order to assure $50 \Omega$ characteristic impedance of a single line, and it was kept unchanged, as well as the values of $t, h$, and $\varepsilon_{r}$, taken in accordance with the parameters of a real printed circuit board. The values of conductor separations were optimized by minimizing the maximum voltage at the MF output. As a result of heuristic search, we obtained the values $s_{1}=200 \mu \mathrm{m}, s_{2}=685 \mu \mathrm{m}$. The voltage waveforms at the input and output of the three-conductor MF with the parameters resulting from the heuristic search are presented in Figure 3. The amplitude of the output voltage was $0.040925 \mathrm{~V}$.

6.2. Optimization by Genetic Algorithm. We used a simple GA implemented in the TALGAT system. The GA was run with typical values (which were not changed further) of the mutation (0.1) and the crossover (0.5) coefficients with a different number of individuals and generations (Table 5). Optimization of $s_{1}$ and $s_{2}$ was performed in the range of $\pm 200 \mu \mathrm{m}$ from the values obtained by heuristic search.

It follows from Section 5.1 that the lowest possible level of amplitude in a three-conductor MF can be achieved by 


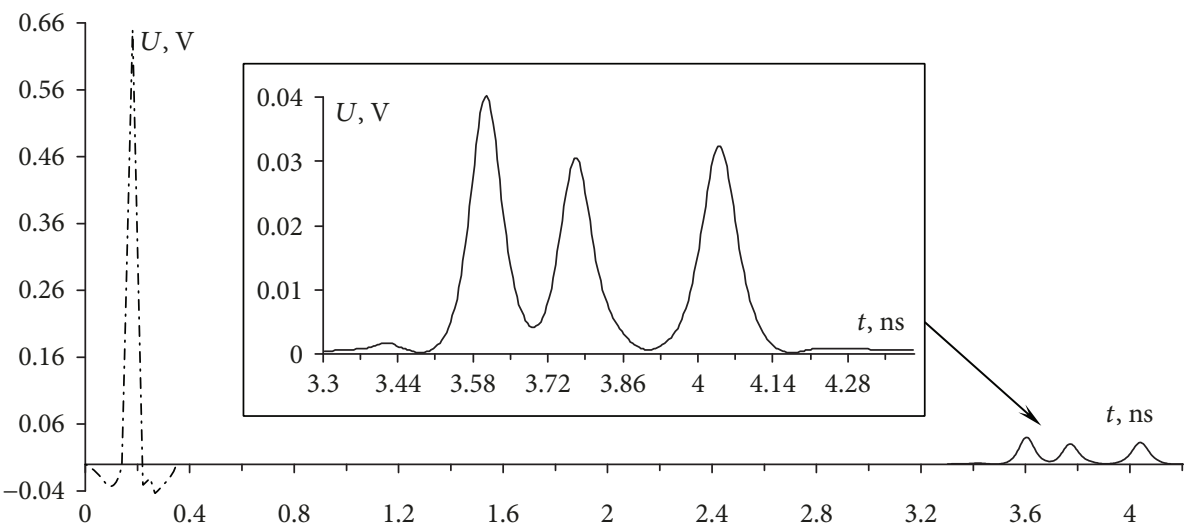

FIGURE 5: Voltage waveforms at the input (---) and output (-) (with enlarged fragment of the signal at the output) of a three-conductor microstrip line MF with the parameters obtained as a result of heuristic search.

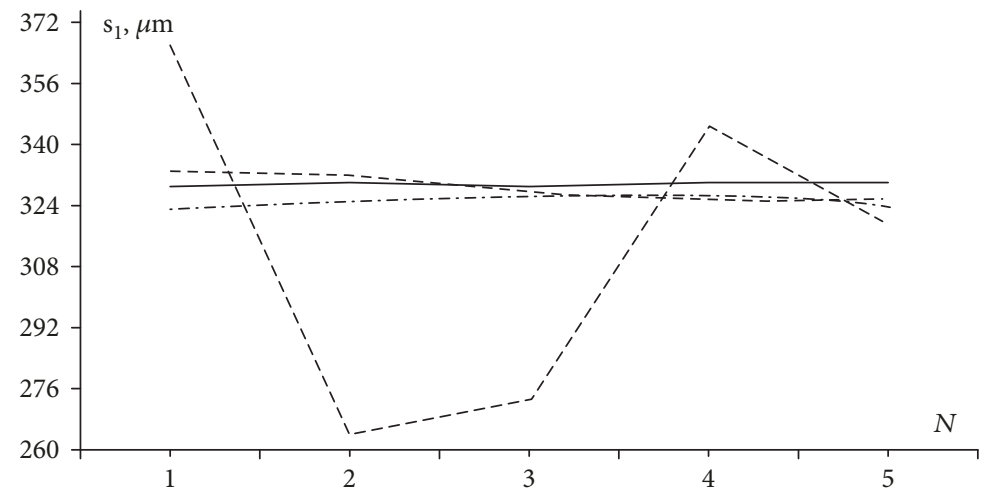

FIgURE 6: The results of GA optimization of $s_{1}$ value for the number of individuals and generations: 3 and $10(--) ; 10$ and $10(\cdots) ; 10$ and 30 (-.-); and 10 and $100(-)$.

equalizing the amplitudes of the decomposition pulses. Meanwhile, Figure 5 shows the possibility to perform further minimization of the pulse amplitudes by their alignment. According to what is stated above, at first, GA was used to optimize $s_{1}$ value only, because the heuristic search revealed that it is the $s_{1}$ that strongly influences the output waveform. The results for the $n$th run of the GA are presented in Table 5, and the graphs for $s_{1}$ are shown in Figure 6.

When the number of individuals and generations was 3 and 10 , respectively (30 calculations), the difference between the extreme values of $s_{1}$ was $38.6 \%$. However, for the number of individuals and generations equal to 10 and 10 , respectively (100 computations), this difference was $2.5 \%$, for 10 and 30 (300 calculations)-1.23\%, and for 10 and 100 (1000 calculations) $-0.3 \%$. As a result, when $s_{1}=330 \mu \mathrm{m}$, we obtained the minimum amplitude of $0.03642 \mathrm{~V}$ (Table 5), which is $12.4 \%$ less than that in the heuristic search.

It follows from what is presented in Table 5 that with the increase in the number of individuals and generations of a GA, the fluctuation of $s_{1}$ value decreases (from $38.6 \%$ to $0.3 \%)$. As a result, for $s_{1}=330 \mu \mathrm{m}$, we obtained the minimum amplitude, which is $12.4 \%$ less than with that in the heuristic search. The voltage waveforms at the input and output of the three-conductor MF with parameters after optimization are presented in Figure 7. It is seen that the amplitudes of the first and second pulses are nearly aligned and the third amplitude is smaller. This could mean the possibility of further reducing the amplitude of the output signal by means of simultaneous optimization of separations $s_{1}$ and $s_{2}$. The results of such optimization for 10 individuals with a change in the number of generations are given in Table 6, and the graphs for $s_{1}$ and $s_{2}$ are shown in Figure 8 . It is seen that with the increase in the number of generations, the fluctuation of the $s_{1}$ and $s_{2}$ values decreases (from $19.3 \%$ and $18.5 \%$ to $0.6 \%$ and $0.7 \%$, resp.).

As a result, when $s_{1}=330 \mu \mathrm{m}$ and $s_{2}=675 \mu \mathrm{m}$, we obtain the maximum possible minimization of the amplitude at the MF output (in this case, the alignment of the first and second pulses) and it is equal to $0.036195 \mathrm{~V}$ (Table 6). It is worth noting that the optimized parameter $s_{1}$ has a value that is equal to the previous one (Table 5) and the change in the parameter $s_{2}$ by $1.5 \%$ (from 685 to $675 \mu \mathrm{m}$ ) helped to align the first and second pulses, thus providing the lowest signal amplitude at the MF output. The voltage waveforms at the input and output of a three-conductor MF with the parameters obtained through optimization using GA are shown in Figure 9.

By optimizing two parameters using GA, we achieved the maximum attenuation of the output signal, obtaining the level of $0.036195 \mathrm{~V}$, which is $0.6 \%$ less than the one obtained 


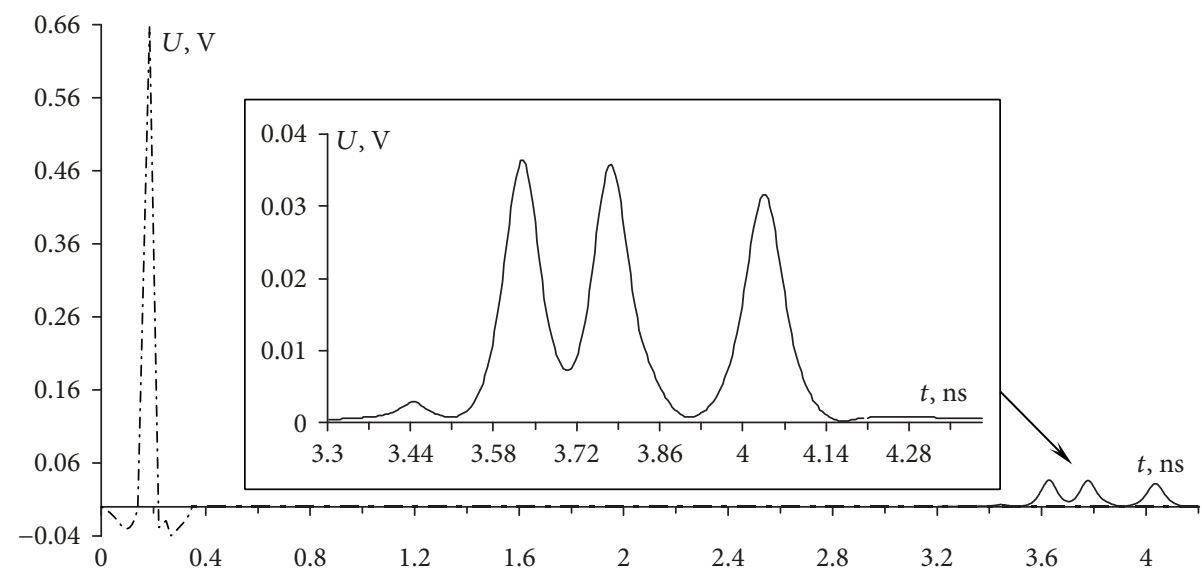

Figure 7: Voltage waveforms at the input (--) and output (-) (with enlarged fragment of the signal at the output) of a three-conductor microstrip line MF after optimization of parameter $s_{1}$ by GA.

TABLE 6: Results of the GA optimization of $s_{1}$ and $s_{2}(\mu \mathrm{m})$ by minimizing the amplitude of the signal $(\max (U(t))(\mathrm{V}))$ at the three-conductor MF output.

\begin{tabular}{|c|c|c|c|c|c|c|c|c|c|}
\hline \multirow{3}{*}{$N$} & \multicolumn{9}{|c|}{ Number of generations } \\
\hline & \multicolumn{3}{|c|}{10} & \multicolumn{3}{|c|}{30} & \multicolumn{3}{|c|}{100} \\
\hline & $s_{1}$ & $s_{2}$ & $\max (U(t))$ & $s_{1}$ & $s_{2}$ & $\max (U(t))$ & $s_{1}$ & $s_{2}$ & $\max (U(t))$ \\
\hline 1 & 389 & 788 & 0.037411 & 350 & 702 & 0.036216 & 330 & 678 & 0.036198 \\
\hline 2 & 328 & 676 & 0.036188 & 329 & 675 & 0.036196 & 329 & 675 & 0.036196 \\
\hline 3 & 335 & 680 & 0.036231 & 334 & 679 & 0.036224 & 330 & 675 & 0.036195 \\
\hline 4 & 359 & 699 & 0.036470 & 339 & 685 & 0.036213 & 331 & 676 & 0.036203 \\
\hline 5 & 326 & 665 & 0.036367 & 328 & 669 & 0.036318 & 331 & 673 & 0.036311 \\
\hline
\end{tabular}

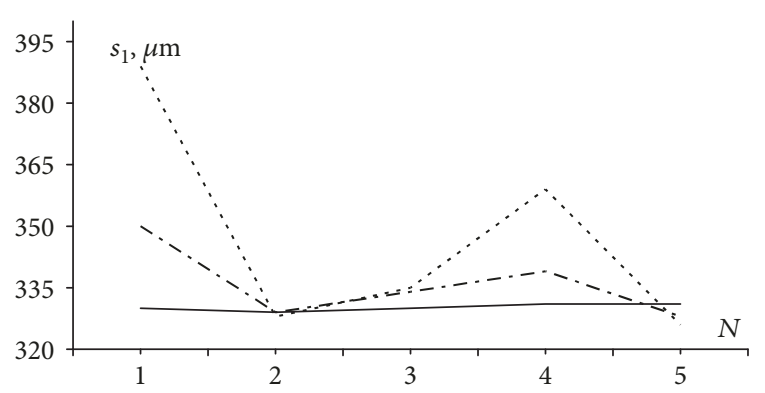

(a)

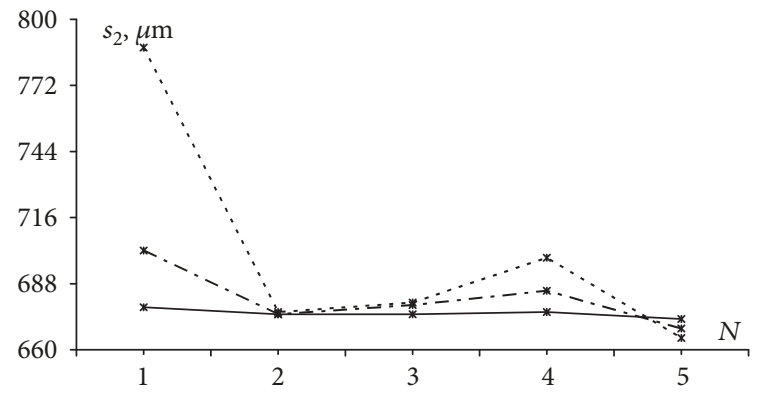

(b)

Figure 8: The results of GA optimization of $s_{1}$ (a) and $s_{2}$ (b) values for 10 individuals and $10(\cdots), 30(-\cdot-)$, and 100 (-) generations.

by optimization of a single parameter and $13 \%$ less than the level obtained by heuristic search. Thus, it is reasonable to use a hybrid model of optimization that should include heuristic search and the use of GA.

6.3. Multicriteria Optimization Using GA. In the previous Sections, we used only one criterion for optimization. As it was noted in Section 5.2, depending on the required aim, one can obtain certain results, that is, when one criterion is reached, another deteriorates. However, it is possible to achieve an optimum, in which all the necessary optimization criteria will be observed as much as required. Thus, it is expedient to formulate a general objective function for the optimization by several criteria and to formulate basic optimization criteria.

6.3.1. General Formulation of the Multicriteria Objective Function. The formulation of a multicriteria objective function $(F)$ implies combining separate criteria to a single problem of minimization or maximization:

$$
F \rightarrow \min \text { or } F \rightarrow \max
$$




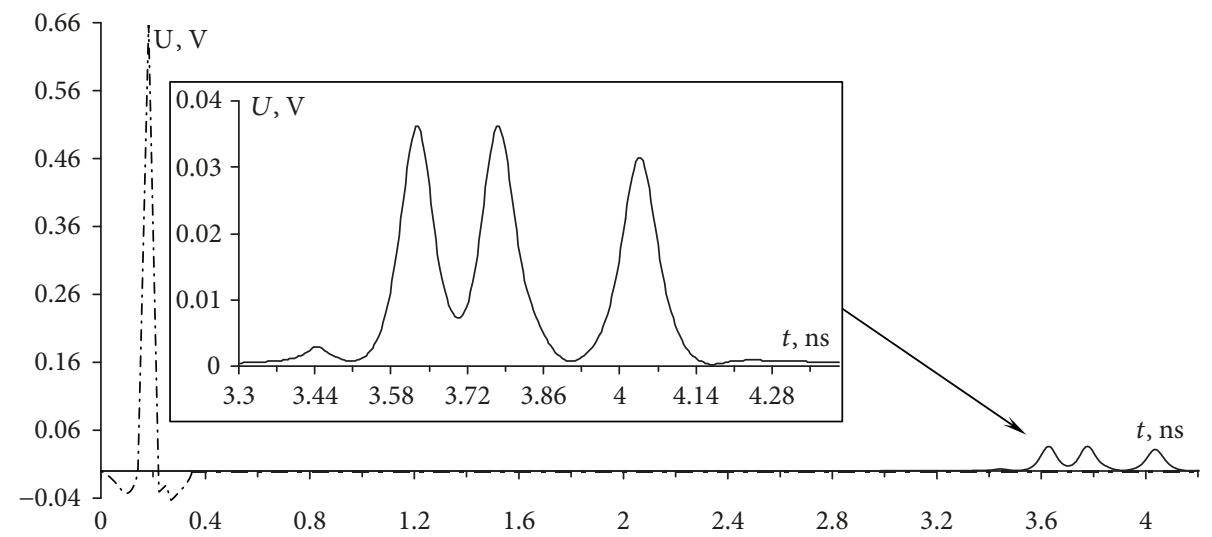

Figure 9: Voltage waveforms at the input (---) and output (-) (with enlarged fragment of the signal at the output) of a three-conductor microstrip line MF after optimization of parameters $s_{1}$ and $s_{2}$ by GA.

For brevity, we will consider the minimization. For example, one can minimize the sum or the maximum of weighted and normalized absolute values of the objective functions that formulate separate criteria:

$$
F=\sum_{i}\left|F_{i}\right| \text { or } F=\max \left|F_{i}\right|
$$

where

$$
F_{i}=M_{i} \frac{f_{i}}{K_{i}},
$$

where $f_{i}$ is the objective function, $K_{i}$ is the normalization constant, $M_{i}$ is the weighting coefficient of the $i$ th criterion, and $i=1,2, \ldots, N_{C}$, where $N_{C}$ is the number of optimization criteria.

The normalization coefficients $K_{i}$ are chosen to be equal to the maximum possible value of the $i$ th objective function so that the value of $f_{i} / K_{i}$ becomes dimensionless and takes values from 0 to 1 during optimization. Moreover, $K_{i}$ must guarantee nonnegative values of $F_{i}$. The significance of the $i$ th criterion is given by the weighting coefficients $M_{i}$. If the criteria are of equal value to the user, then these coefficients are the same and can be given as

$$
M_{i}=\frac{1}{N_{C}} .
$$

Optimization can be performed according to various criteria. Amplitude and time criteria are relevant to the electrical characteristics of multiconductor MFs. They are discussed in detail in the following sections.

6.3.2. Amplitude Criteria. The most important criteria for optimization of an MF are amplitude ones. They can be considered in the time and frequency domains. It is useful to analyze voltage waveforms $U(t)$ at the MF output to provide protection against the exciting ultrashort pulse of electromotive force $E(t)$. Therefore, let us consider the amplitude criteria in the time domain. On the basis of $U(t)$, five norms used to evaluate the effectiveness of ultrashort pulses impact on different (according to specificity of the response to the impact) equipment [2]. Using these norms, we can formulate expressions for $f_{i}$ and $K_{i}$.

(1) For the circuit upset, as well as electric breakdown or arc-over effects, the maximum magnitude of the $U(t)$ is important:

$$
\begin{aligned}
f_{1} & =\max |U(t)|, \\
K_{1} & =\max |E(t)| .
\end{aligned}
$$

(2) For component arcing, as well as the circuit upset, the maximum magnitude of the $U(t)$ change rate is important:

$$
\begin{gathered}
f_{2}=\max \left|\frac{\partial U(t)}{\partial t}\right|, \\
K_{2}=\max \left|\frac{\partial E(t)}{\partial t}\right| .
\end{gathered}
$$

(3) For dielectric puncture, the maximum magnitude of the integral of the $U(t)$ is important:

$$
\begin{gathered}
f_{3}=\max \left|\int_{0}^{t} U(t) d t\right|, \\
K_{3}=\max \left|\int_{0}^{t} E(t) d t\right| .
\end{gathered}
$$

(4) For equipment damage, the integral of the $U(t)$ magnitude is important:

$$
\begin{aligned}
f_{4} & =\int_{0}^{\infty}|U(t)| d t, \\
K_{4} & =\int_{0}^{\infty}|E(t)| d t .
\end{aligned}
$$


TABLE 7: Optimized parameters of MF for $N=3$, their ranges, and values after optimization with different weighing coefficients.

\begin{tabular}{lcccc}
\hline Parameter & Range & $M_{1}=M_{2}=M_{3}=M_{4}=0.25$ & $M_{1}=M_{2}=M_{3}=0.3, M_{4}=0.1$ & $M_{1}=0.1, M_{2}=M_{3}=M_{4}=0.3$ \\
\hline$t, \mu \mathrm{m}$ & $10-200$ & 174 & 176 & 200 \\
$h, \mu \mathrm{m}$ & $200-2000$ & 995 & 200 & 201 \\
$s_{1}, \mu \mathrm{m}$ & $1-1000$ & 10 & 4 & 3 \\
$s_{2}, \mu \mathrm{m}$ & $1-1000$ & 115 & 48 & 44 \\
\hline
\end{tabular}

(5) For component burnout, the square root of the integral of the square of the $U(t)$ magnitude is important:

$$
\begin{aligned}
f_{5} & =\left\{\int_{0}^{\infty}|U(t)|^{2} d t\right\}^{1 / 2}, \\
K_{5} & =\left\{\int_{0}^{\infty}|E(t)|^{2} d t\right\}^{1 / 2} .
\end{aligned}
$$

6.3.3. Time Criteria. Time criteria are important for preventing pulses that increase the maximum voltage at the MF output with increasing duration of the exciting ultrashort pulse. In contrast to amplitude ones, time criteria may not require costly computation of the response, since it is enough to calculate only the per-unit-length delays. We consider three types of time criteria.

The minimum-time criterion and the maximum-time criterion are associated with the expansion of the pulse time range at the MF output. The time interval criterion is related to the alignment of time intervals. Note that, in time criteria, the values of a per-unit-length delay are sorted out in ascending order.

The minimum-time criterion makes a per-unit-length delay of the first pulse $\left(\tau_{\min }\right)$ as short as possible, that is, as determined by the light velocity in vacuum:

$$
\begin{aligned}
f_{6} & =\tau_{\text {min }}-\frac{1}{c}, \\
K_{6} & =\frac{\sqrt{\varepsilon_{r \max }}-1}{\mathrm{c}} .
\end{aligned}
$$

The maximum-time criterion makes a per-unit-length delay of the last pulse $\left(\tau_{\max }\right)$ as long as possible, that is, as determined by the light velocity in dielectric with the maximum value of the relative dielectric permittivity $\left(\varepsilon_{r \max }\right)$ :

$$
\begin{aligned}
f_{7} & =\frac{\sqrt{\varepsilon_{r \max }}}{\mathrm{c}}-\tau_{\max }, \\
K_{7} & =\frac{\sqrt{\varepsilon_{r \max }}-1}{\mathrm{c}} .
\end{aligned}
$$

To expand the time range in both directions, these criteria must be used together. They are applicable to an MF with any value of $N$.

The time interval criterion is important when $N>2$. It is used to equalize time intervals between the pulses at the MF output. It allows increasing the duration of the exciting ultrashort pulse, which will be decomposed at the MF output completely. For the values of per-unit-length delays which are sorted out in an ascending order, and based on the deviation of the current values of the per-unit-length delays of the intermediate modes from the values according to the uniform time intervals between the pulses, we obtain

$$
\begin{aligned}
f_{8} & =\max \left|\tau_{i}-\left(\tau_{\min }+(i-1) \cdot \Delta\right)\right|, \quad i=2, \ldots, N-1, \\
K_{8} & =\frac{\sqrt{\varepsilon_{r \max }}-1}{\mathrm{c}}
\end{aligned}
$$

where

$$
\Delta=\frac{\tau_{\max }-\tau_{\min }}{N-1}
$$

where $\tau_{i}$ is the value of a per-unit-length delay of the $i$ th pulse.

6.4. Multicriteria GA Optimization of $M F$ with $N=3$. To test the theory, a three-conductor microstrip MF was optimized by GA. We used the multicriteria objective function that combines one amplitude (14) and three (19), (20), and (21) time criteria with equal weighting coefficients for $N=3$ :

$$
\begin{aligned}
F= & M_{1} \cdot\left(\frac{\max (U(t))}{\max (E(t))}\right)+M_{2} \cdot\left(\frac{\tau_{1}-(1 / \mathrm{c})}{\left(\sqrt{\varepsilon_{r \max }}-1\right) / \mathrm{c}}\right) \\
& +M_{3} \cdot\left(\frac{\left(\sqrt{\varepsilon_{r \max }} / \mathrm{c}\right)-\tau_{3}}{\left(\sqrt{\varepsilon_{r \max }}-1\right) / c}\right)+M_{4} \cdot\left(\frac{\left|2 \tau_{2}-\tau_{1}-\tau_{3}\right|}{\left(\sqrt{\varepsilon_{r \max }}-1\right) / \mathrm{c}}\right) .
\end{aligned}
$$

The GA parameters were chosen (and further remained unchanged) as follows: number of individuals was 50; number of generations was 100 .

The MF was optimized for the following parameters: $w=1000 \mu \mathrm{m}, \varepsilon_{r}=5, l=60 \mathrm{~cm}$, and $R=50 \Omega$. The value of $w$ was optimized in order to provide $50 \Omega$ characteristic impedance of a single line, and it was unchanged, as well as the value of $\varepsilon_{r}$. The values of $t, h$, and $s_{1}, s_{2}$ were optimized by the multicriteria objective function (23). The optimized parameters, their ranges, and values after optimization with different weighing coefficients are given in Table 7. For $M_{1}=M_{2}=M_{3}=M_{4}=0.25$, the amplitude was minimized to $0.03066 \mathrm{~V}$, the equalized values of $\Delta \tau_{i}$ were 1.24317 and $1.24372 \mathrm{~ns} / \mathrm{m}$, so their difference was minimized to $0.00055 \mathrm{~ns} / \mathrm{m}$, and the value $\left(\tau_{\max }-\tau_{\min }\right)$ was maximized to $2.48689 \mathrm{~ns} / \mathrm{m}$. The voltage waveforms at the input and output of the MF with the parameters after the GA optimization are presented in Figure 10. Thus, the attenuation 


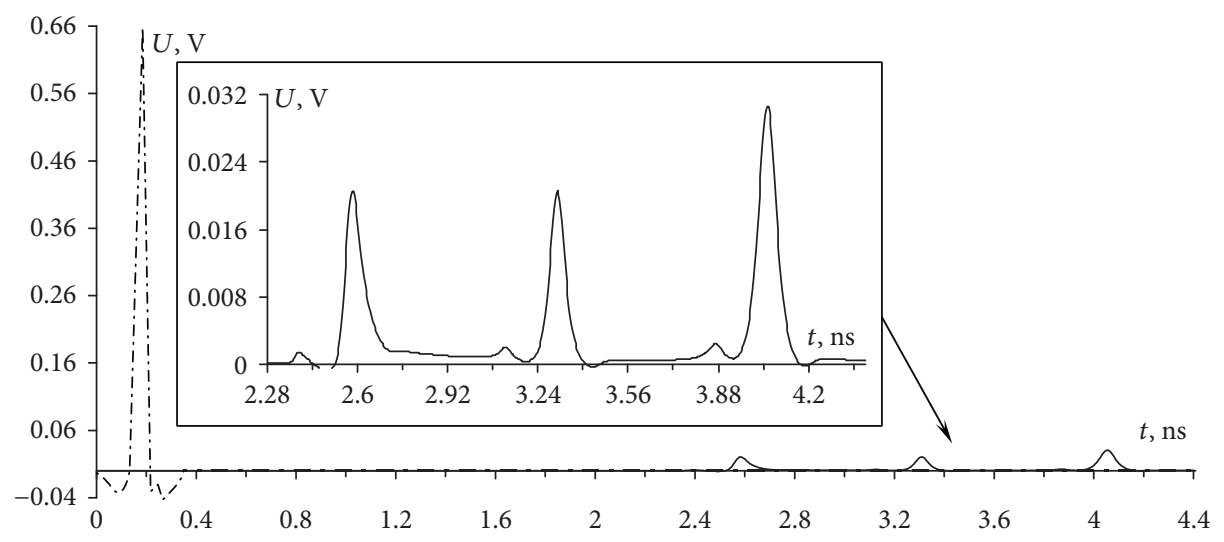

Figure 10: Voltage waveforms at the input (---) and output (-) (with enlarged fragment of the signal at the output) of a three-conductor microstrip line MF after four-criterion GA optimization.

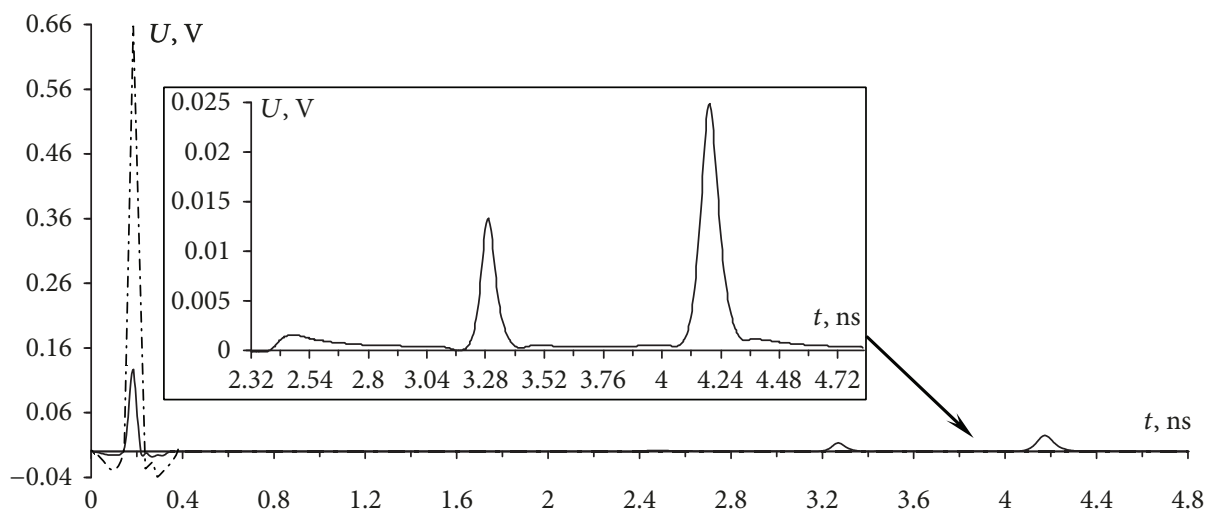

Figure 11: Voltage waveforms at the input (---) and output (-) (with enlarged fragment of the signal at the output) of a three-conductor microstrip line MF after the four-criterion GA optimization with the objective function (23), when time interval criterion is less important.

factor of 21.4 is possible for ultrashort pulse with the duration of less than $0.6 \mathrm{~ns}$.

It is also useful to perform multicriteria optimization using 4 criteria (23), but with different weighing coefficients. Suppose that for a designer the amplitude and the maximumtime and minimum-time criteria are essential, while the time interval criterion is less important. Table 7 shows the values of the parameters after optimization for $M_{1}=M_{2}=M_{3}=$ 0.3 and $M_{4}=0.1$. In this case, the amplitude was minimized to $0.0247953 \mathrm{~V}$, the equalized values of $\Delta \tau_{i}$ were 1.50777 and $1.38147 \mathrm{~ns} / \mathrm{m}$, so their difference was minimized to $0.1263 \mathrm{~ns} / \mathrm{m}$, and the value $\left(\tau_{\max }-\tau_{\min }\right)$ was maximized to $2.88924 \mathrm{~ns} / \mathrm{m}$. The voltage waveforms at the MF input and output with parameters after the GA optimization are presented in Figure 11. For this once suppose that for a designer the maximum-time, minimum-time and time-interval criteria are essential, while the amplitude criterion is less important. Then we can define $M_{1}=0.1$ and $M_{2}=M_{3}=M_{4}=0.3$. Table 7 shows the values of the parameters after this optimization. In this case, the amplitude was minimized to $0.0249575 \mathrm{~V}$, the equalized values of $\Delta \tau_{i}$ were 1.501 and $1.505 \mathrm{~ns} / \mathrm{m}$, so their difference was minimized to $0.004 \mathrm{~ns} / \mathrm{m}$, and the value $\left(\tau_{\max }-\tau_{\min }\right)$ was maximized to $3.00546 \mathrm{~ns} / \mathrm{m}$. The voltage waveforms at the input and output of the MF with parameters after the GA optimization are presented in Figure 12.

6.5. Multicriteria Optimization of $M F$ with $N=4$ with Respect to the Criterion of Matching. Previously, the matching of tract was neglected for $N>3$. In addition, multicriteria optimization of a microstrip MF for $N>3$ by GA has not been performed before. However, it is relevant, since the increase in the number of conductors, in general, improves the characteristics of the MF. Thus, multicriteria optimization of a four-conductor microstrip MF with respect to the matching criterion is useful.

6.5.1. Formulation of the Matching Criterion. One of the important criteria for optimizing an MF is the matching criterion. This criterion is important in order to minimize the reflection of useful high-frequency signals from the MF.

The condition for matching the two coupled lines by resistances $R$ at their ends is defined as the geometric mean value of the impedances of the even and odd modes [15]:

$$
R=\sqrt{Z_{e} Z_{o}}
$$




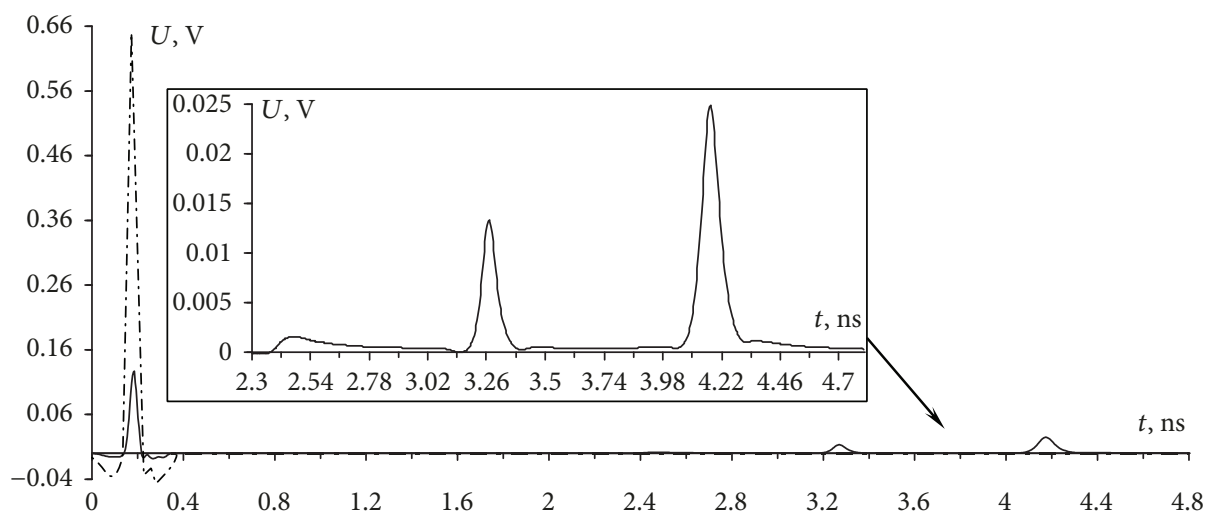

FIgURe 12: Voltage waveforms at the input (--) and output (-) (with enlarged fragment of the signal at the output) of a three-conductor microstrip line MF after the four-criterion GA optimization with the objective function (23), when amplitude criterion is less important.

In multiconductor transmission lines, the number of propagating modes is equal to the number of conductors $(N)$. From the eigenvalues of the impedance matrix of the line, it is possible to determine the mode impedances. The subsequent stages of determining the matching condition are apparently possible on the basis of the theory of multiconductor transmission lines, but are not clear for authors yet. Meanwhile, by analogy with a matched single transmission line, to match a multiconductor transmission line, we can use the condition of equality of the signal amplitude at the beginning of the line $U_{\mathrm{IN}}(t)$ and half the electromotive force of the signal source $E(t)$. Then, after simplification, we get

$$
\begin{aligned}
f_{9} & =\left|\max (E(t))-2 \max \left(U_{\mathrm{IN}}(t)\right)\right|, \\
K_{9} & =\max |E(t)| .
\end{aligned}
$$

This criterion must be used for $N>2$. However, it requires the calculation of the time response at the beginning of the line, but without significant additional costs if we perform the frequency domain analysis [16].

6.5.2. Optimization of the Four-Conductor MF with Respect to Matching Criterion. A multicriteria objective function that combines one amplitude, three time, and a matching criterion (obtained for $N=4$ ) as well looks (with weighing coefficients equal to 1) like

$$
\begin{aligned}
F= & \frac{\max (U(t))}{\max (E(t))}+\frac{\tau_{1}-(1 / \mathrm{c})}{\left(\sqrt{\varepsilon_{r \max }}-1\right) / \mathrm{c}}+\frac{\left(\sqrt{\varepsilon_{r \max }} / \mathrm{c}\right)-\tau_{4}}{\left(\sqrt{\varepsilon_{r \max }}-1\right) / \mathrm{c}} \\
& +\frac{\max \left(\left|\tau_{2}-\left(\left(\tau_{4}+2 \tau_{1}\right) / 3\right)\right|,\left|\tau_{3}-\left(\left(\tau_{1}+2 \tau_{4}\right) / 3\right)\right|\right)}{\left(\sqrt{\varepsilon_{r \max }}-1\right) / \mathrm{c}} \\
& +\frac{\left|\max (E(t))-2 \max \left(U_{\mathrm{IN}}(t)\right)\right|}{\max |E(t)|} .
\end{aligned}
$$

The MF was optimized for the following parameters: $w=180 \mu \mathrm{m}, \varepsilon_{r}=5, l=60 \mathrm{~cm}$, and $R=50 \Omega$. The value of $w$ was unchanged, as well as the value of $\varepsilon_{r}$. The values of $t, h$, and $s_{1}, s_{2}$, and $s_{3}$ were optimized by the five-
TABle 8: Optimized parameter of the MF for $N=4$, their ranges, and values after optimization with respect to the matching criterion with equal weighing coefficients.

\begin{tabular}{lcc}
\hline Parameter & Range & Value \\
\hline$t, \mu \mathrm{m}$ & $10-200$ & 35 \\
$h, \mu \mathrm{m}$ & $200-2000$ & 501 \\
$s_{1}, \mu \mathrm{m}$ & $1-1000$ & 8 \\
$s_{2}, \mu \mathrm{m}$ & $1-1000$ & 23 \\
$s_{3}, \mu \mathrm{m}$ & $1-1000$ & 390 \\
\hline
\end{tabular}

criterion objective function (26). The optimized parameters, their ranges, and values after optimization are given in Table 8. As a result of optimization, the amplitude was minimized to $0.0188094 \mathrm{~V}$, the equalized values of $\Delta \tau_{i}$ were $0.53008,0.52194$, and $0.52134 \mathrm{~ns} / \mathrm{m}$, differing by $2 \%$, and the value $\left(\tau_{\max }-\tau_{\min }\right)$ was maximized to $1.57336 \mathrm{~ns} / \mathrm{m}$. In addition, the amplitude at the input MF input was $0.323928 \mathrm{~V}$, which is 2.03 times less than the EMF of the source $(0.657608 \mathrm{~V})$, thereby ensuring the matching. The voltage waveforms at the input and output of the four-conductor MF with parameters after the GA optimization are presented in Figure 13.

Thus, in this section, we proposed and formulated a matching criterion for optimizing a multiconductor MF. Then, we obtained the five-criterion objective function for a four-conductor microstrip MF followed by the optimization of five parameters using this function. At the output of the MF, well matched to the $50 \Omega$ path, we obtained the signal with the amplitude of $0.0188094 \mathrm{~V}$ and close differences in the per-unit-length delays of adjacent pulses. Thus, the attenuation factor of 34.9 is possible not only for the ultrashort pulse with the duration of $65 \mathrm{ps}$ considered above, but also for much longer pulses (about 100-200 ps).

Previously, we obtained a three-conductor MF for $s_{1}=10 \mu \mathrm{m}$ and $s_{2}=115 \mu \mathrm{m}$ with a maximum output amplitude of $0.03066 \mathrm{~V}$. The optimization was carried out with respect to four criteria and four parameters in the same range. The amplitude at the MF output of this Section is $63 \%$ less. 


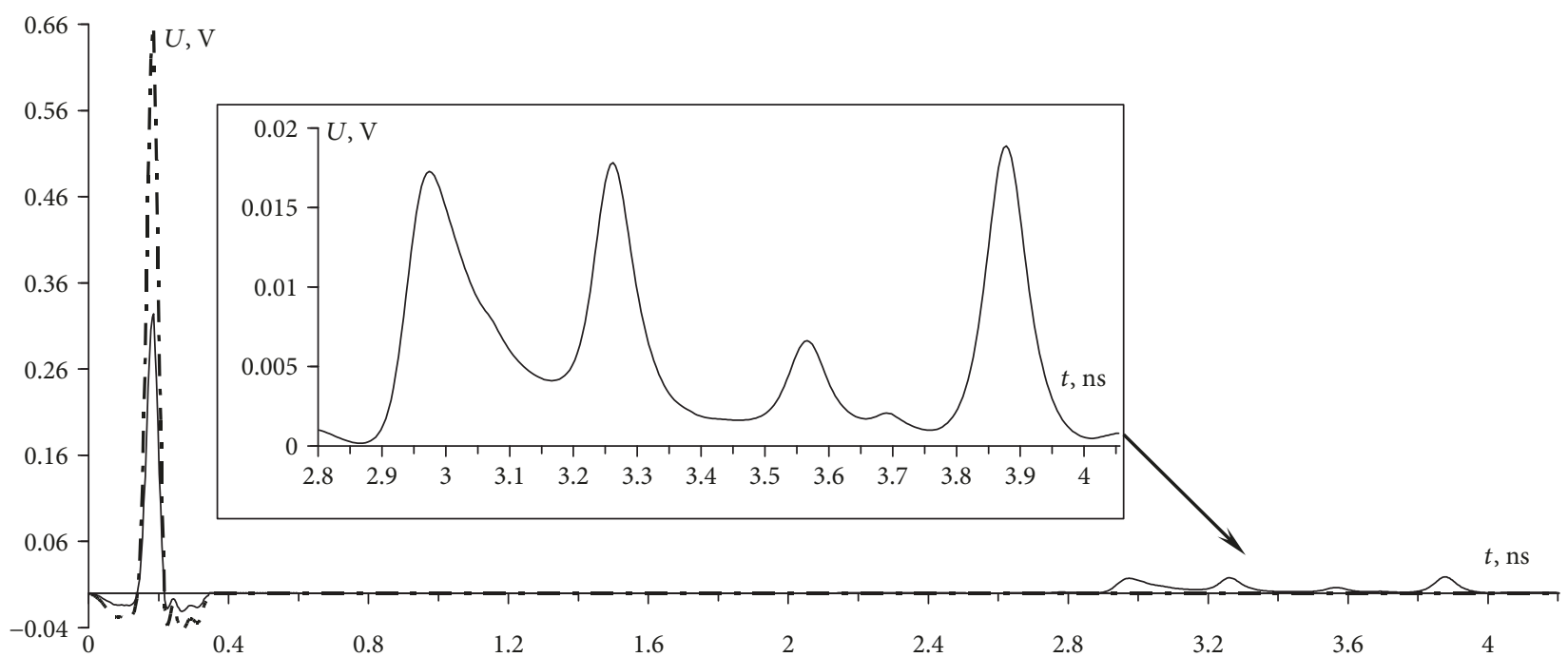

Figure 13: Waveforms of the EMF (--) and input (-) and output (-) voltages (with enlarged fragment of the voltage waveform at the output) of a four-conductor microstrip line MF with the parameters obtained as a result of five-criterion GA optimization of five parameters.

In conclusion, we note the methodological significance of the results of this Section:

(i) A universal five-criterion objective function is suitable for optimizing any four-conductor MF.

(ii) The time interval criterion for a four-conductor MF is obtained in an analytical form.

(iii) The equalization of time intervals between the decomposed pulses is approved.

These results can be successfully employed to optimize one of the new and advancing versions of a fourconductor MF called a mirror-symmetric MF [17]. In such $\mathrm{MF}$, the equality of the pulse amplitudes is relatively easy to obtain, while equalizing the intervals between pulses may require optimization.

\section{Conclusion}

Thus, for the first time, the work presents the results of a systematic study of the possibility (both separate and simultaneous) to optimize multiconductor MFs with respect to different criteria. The formulation of the basic (electrical) optimization criteria for a MF has been performed, and analytical expressions have been proposed, which can significantly accelerate the calculation of the objective function. The amplitude and time criteria for optimizing an MF in analytical form are formulated, and a general multicriteria objective function is obtained, which allows, in the long term, to use any optimization methods and obtain higher MF characteristics. The article presents the results of optimization by heuristic search and a simple GA, and, to improve the optimization, we have formed a hybrid model consisting of heuristic search and the GA, with the formulated criteria being applicable to an MF with any $N$.
Note that it is useful to consider such MFs in the frequency domain and to optimize them with respect to the relevant criteria. So, for example, it is important to use an $\mathrm{MF}$ to protect against interference at a given frequency, as well as to provide the necessary bandwidth of a useful signal. In that case, it is possible to formulate some additional criteria that take into account the requirements in the frequency domain. In addition, it is essential to take into account the requirements to minimize mass dimensions (e.g., to protect space vehicles) as well as the cost (for large series production).

\section{Data Availability}

The data used to support the findings of this study are available from the corresponding author upon request.

\section{Conflicts of Interest}

The authors declare that there is no conflict of interests regarding the publication of this paper.

\section{Acknowledgments}

The authors thank the reviewers for valuable comments which have made it possible to considerably improve the article. This research was supported by the Ministry of Education and Science of the Russian Federation (Project 8.9562.2017/8.9).

\section{References}

[1] Z. M. Gizatullin and R. M. Gizatullin, "Investigation of the immunity of computer equipment to the power-line electromagnetic interference," Journal of Communications Technology and Electronics, vol. 61, no. 5, pp. 546-550, 2016. 
[2] N. Mora, F. Vega, G. Lugrin, F. Rachidi, and M. Rubinstein, Study and Classification of Potential IEMI Sources, System Design and Assessment Notes, Note 41, 2014.

[3] A. M. Zabolotsky and A. T. Gazizov, "Simulation of ultrawide band pulse propagation in asymmetrical modal filter for power network protection," International Journal of Circuits, Systems and Signal Processing, vol. 9, pp. 68-74, 2015.

[4] A. T. Gazizov, A. M. Zabolotsky, and T. Rashitovich Gazizov, "UWB pulse decomposition in simple printed structures," IEEE Transactions on Electromagnetic Compatibility, vol. 58, no. 4, pp. 1136-1142, 2016.

[5] M. Reformat, E. Kuffel, D. Woodford, and W. Pedrycz, "Application of genetic algorithms for control design in power systems," IEE Proceedings - Generation, Transmission and Distribution, vol. 145, no. 4, pp. 345-354, 1998.

[6] S. K. Goudos, C. Kalialakis, and R. Mittra, "Evolutionary algorithms applied to antennas and propagation: a review of state of the art," International Journal of Antennas and Propagation, vol. 2016, Article ID 1010459, 12 pages, 2016.

[7] A. O. Belousov, A. M. Zabolotsky, and T. T. Gazizov, "Experimental confirmation of the modal filtration in four- and five-conductor microstrip lines," in 2017 18th International Conference of Young Specialists on Micro/Nanotechnologies and Electron Devices (EDM), pp. 46-49, Erlagol, Russia, 2017.

[8] A. O. Belousov, T. R. Gazizov, and A. M. Zabolotsky, "Maximization of duration of ultrashort pulse that is completely decomposed in multiconductor modal filters," in 2016 International Siberian Conference on Control and Communications (SIBCON), pp. 1-4, Moscow, Russia, 2016.

[9] A. O. Belousov, A. M. Zabolotsky, and T. T. Gazizov, "Optimization of parameters of multiconductor modal filters for protection against ultrashort pulses," in 2016 17th International Conference of Young Specialists on Micro/Nanotechnologies and Electron Devices (EDM), pp. 67-70, Erlagol, Russia, 2016.

[10] A. O. Belousov, A. M. Zabolotsky, and T. R. Gazizov, "Optimization of parameters of multiconductor modal filters for protection against ultrashort pulses," in Materials of the all-russian scientific and technical conference "Sovremennye problemy radioehlektroniki», pp. 392-396, Krasnoyarsk, Russia, 2016.

[11] A. O. Belousov, T. T. Gazizov, and T. R. Gazizov, "Multicriteria optimization of four-conductor modal filter by genetic algorithms," in 2017 International Multi-Conference on Engineering, Computer and Information Sciences (SIBIRCON), pp. 445-448, Novosibirsk, Russia, 2017.

[12] A. R. Djordjevic, R. M. Biljie, V. D. Likar-Smiljanic, and T. K. Sarkar, "Wideband frequency-domain characterization of FR-4 and time-domain causality," IEEE Transactions on Electromagnetic Compatibility, vol. 43, no. 4, pp. 662-667, 2001.

[13] G. L. Matthaei and G. C. Chinn, “Approximate calculation of the high-frequency resistance matrix for multiple coupled lines," in 1992 IEEE Microwave Symposium Digest MTT-S, pp. 1353-1354, Albuquerque, NM, USA, 1992.

[14] S. P. Kuksenko, T. R. Gazizov, A. M. Zabolotsky et al., "New developments for improved simulation of interconnects based on method of moments," in Proceedings of the 2015 International Conference on Modeling, Simulation and Applied Mathematics, pp. 293-301, Phuket, Thailand, August 2015.
[15] E. M. T. Jones, "Coupled-strip-transmission-line filters and directional couplers," IEEE Transactions on Microwave Theory and Techniques, vol. 4, no. 2, pp. 75-81, 1956.

[16] R. Achar and M. S. Nakhla, "Simulation of high-speed interconnects," Proceedings of the IEEE, vol. 89, no. 5, pp. 693-728, 2001.

[17] A. M. Zabolotsky, "Application of reflective symmetry for modal filtration improvement," Dokladi Tomsk. gos. un-ta sist. upr. i radioelectroniki, vol. 2, no. 36, pp. 41-44, 2015. 


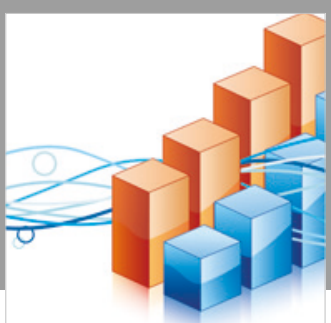

Advances in

Operations Research

\section{-n-m}
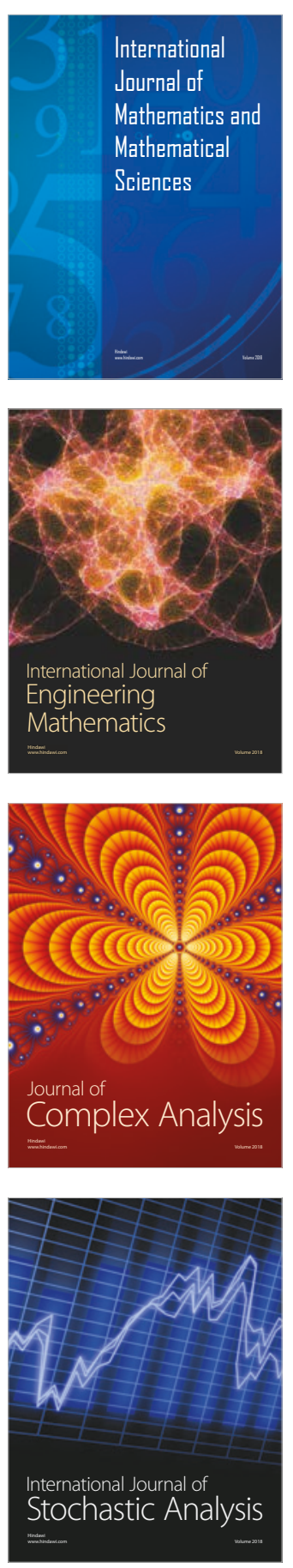
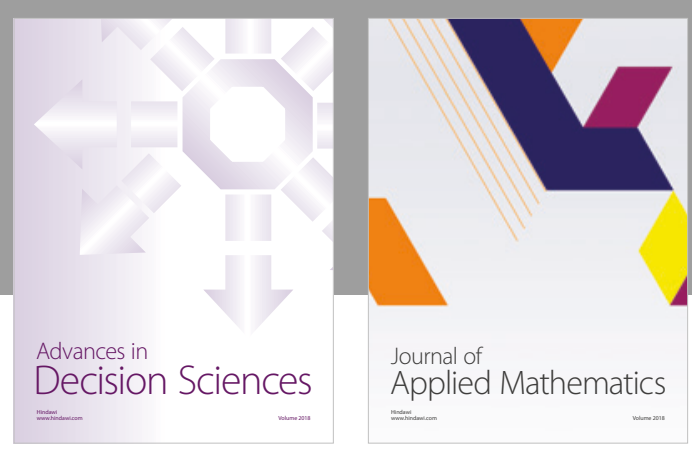

Journal of

Applied Mathematics
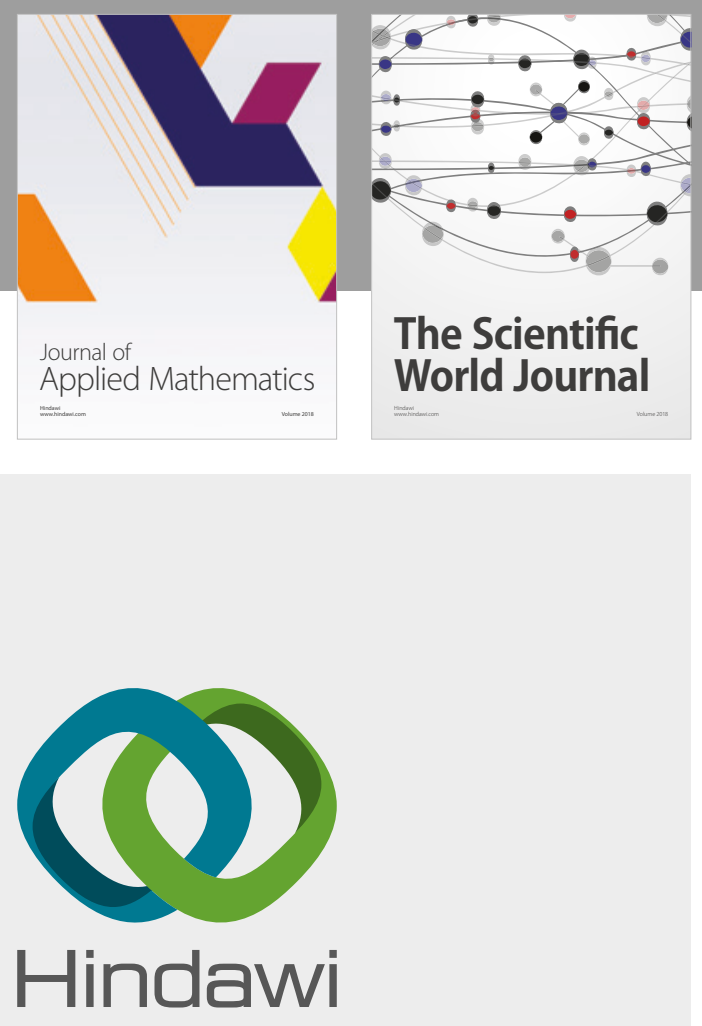

Submit your manuscripts at

www.hindawi.com

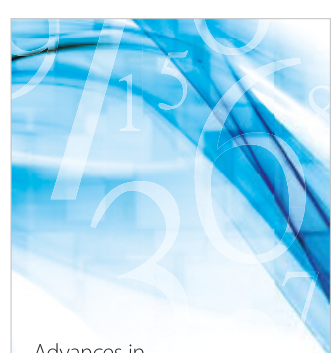

Advances in
Numerical Analysis
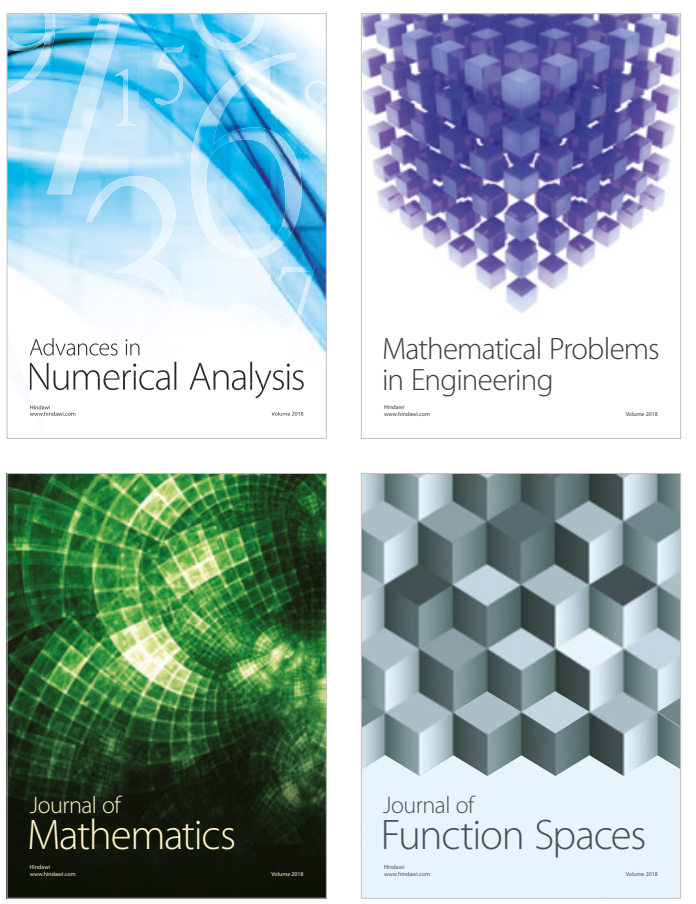

Mathematical Problems in Engineering

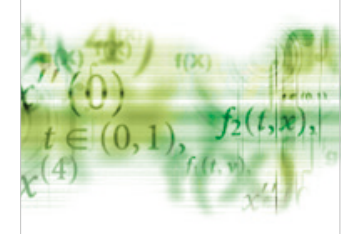

International Journal of

Differential Equations

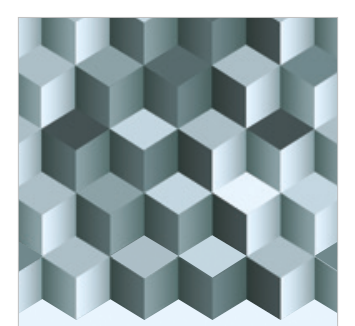

Journal of

Function Spaces
The Scientific

World Journal

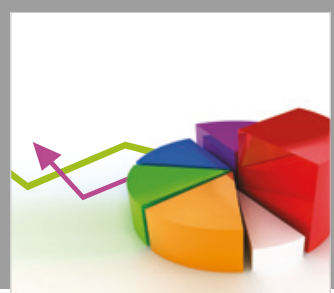

Journal of

Probability and Statistics
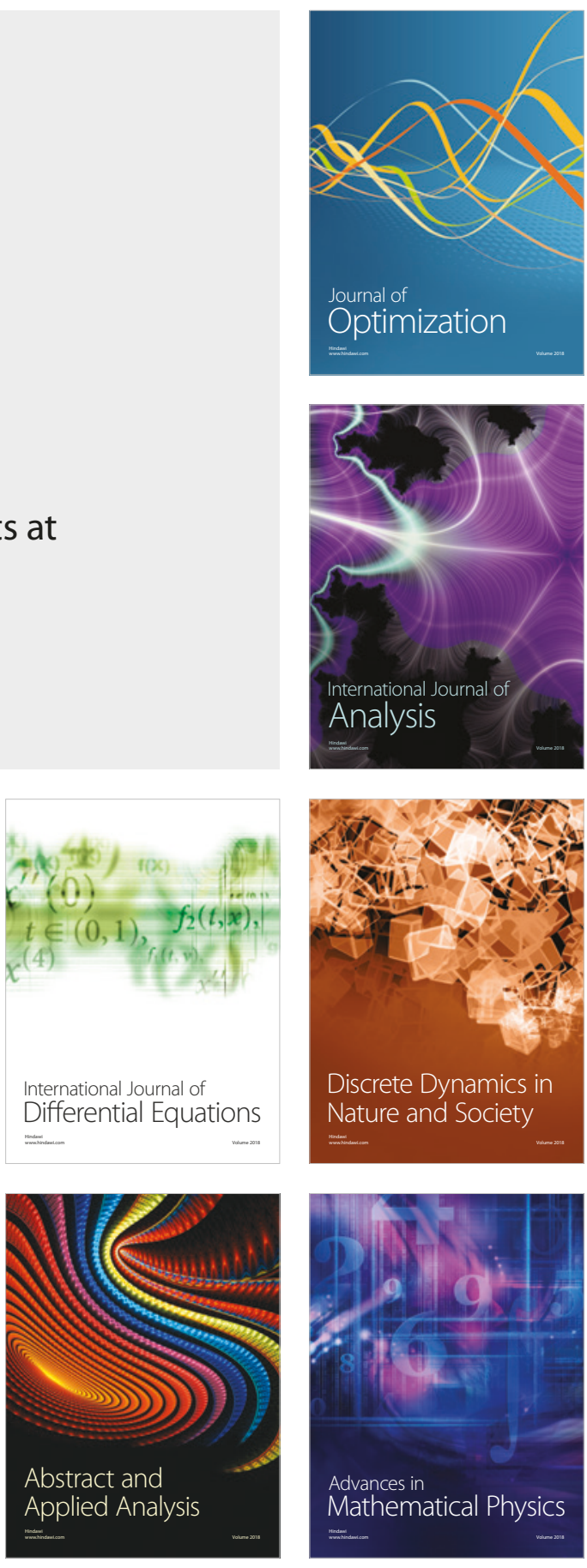